\title{
Modelling Temperature Effect on (Transverse Electric) TE Mode Shape of Optical Silica Waveguide
}

\author{
Indrawan Arifianto ${ }^{*}$, Muhammad Rangga Hadisiswoyo, Ary Syahriar \\ Department of Electrical Engineering, Faculty of Science and Engineering, Al Azhar University of Indonesia, Indonesia
}

Copyright $(2019$ by authors, all rights reserved. Authors agree that this article remains permanently open access under the terms of the Creative Commons Attribution License 4.0 International License

\begin{abstract}
Theoretical approach to study the influence of temperature of the effective refractive index value for asymmetric nonlinear optical waveguides was developed. Waveguide structures are equipped with linear cladding and substrate. Numerical modeling and calculations were performed to measure the sensitivity of the effective refractive index core thickness for transverse electrical (TE) mode and transversal magnetic (TM) mode. Based on the results, which affect the waveguide refractive index value, we can also adjust the waveguide by varying the temperature value. The waveguide structure is completed by linear cladding and substrate.
\end{abstract}

Keywords Modelling, Temperature, Propagation Constant, Effective Refractive Index, Slab Waveguide, Asymmetric Waveguide

\section{Introduction}

Temperature is an important issue in developing optoelectronic devices. Environmental temperature variations cause temperature incompatibility of different components in photonic devices. Temperature parameters cause changes in optical parameters. This is because of the compatibility of the refractive index and the length of the optical path from the waveguide. Parameters of optical variation, such as wavelength and effective refractive index can be changed so that the results can be used as a measure of changes in environmental temperature. A waveguide works as a transducer that converts perturbations such as temperature, voltage, strain, rotation or electric current and magnetic changes to be appropriate to radiation [1].

The temperature sensitivity of the effective refractive index TE mode of three linear media waveguides has been considered by Huang [8]. The focus of this research is on the effect of the asymmetric waveguide sensor temperature by looking at the TE and TM modes. Part 2 describes basic theory, the configuration of a waveguide, and to solve the wave equation and mode equation from the waveguide.
The focus of section 3 is to describe the methodology research. In section 4, attention will be directed to the thermal sensitivity of the effective refractive index. Section 5 is dedicated to Results and discussion, followed by conclusions.

\section{Basic Theory}

As shown in Figure 1, the basic structure of the waveguide in this analysis consists of three layers: substrate, core and cladding. The core is an infinitely large planar dielectric film with a thickness of the order of wavelengths. The core refractive index is considered higher than the refractive index of the surrounding media. The surrounding media are linear media and nonlinear cladding. The cladding refractive index profile is assumed to have Kerr like intensity dependence expressed as $\varepsilon_{c l}=\varepsilon_{0}+\alpha\left|E_{y}\right|^{2}$, where $\varepsilon_{0}$ is the linear relative permittivity and $\alpha$ is thenonlinear coefficient

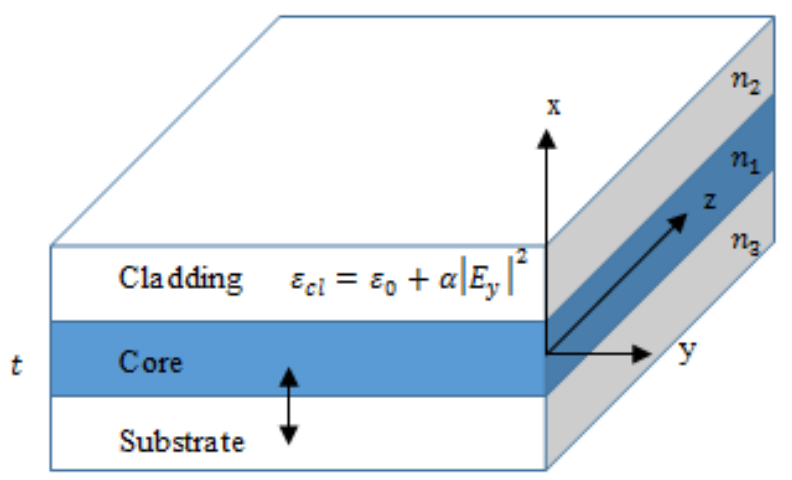

Figure 1. Asymmetric Waveguide

The direction of light propagation is parallels to the $\mathrm{z}$-axis, confined in the $\mathrm{x}$ direction within the central core, and the variation in the y direction is ignored. In this study, the new structure of the waveguide is investigated for both TE and TM fields.

Depending on whether a total internal reflection occurs 
at the core-substrate or/and core-cover interfaces, there are at least three types of modes that may be supported by waveguide. They are guided modes, substrate radiation modes and superstrate-cover radiation modes as indicated in Figure 2 below

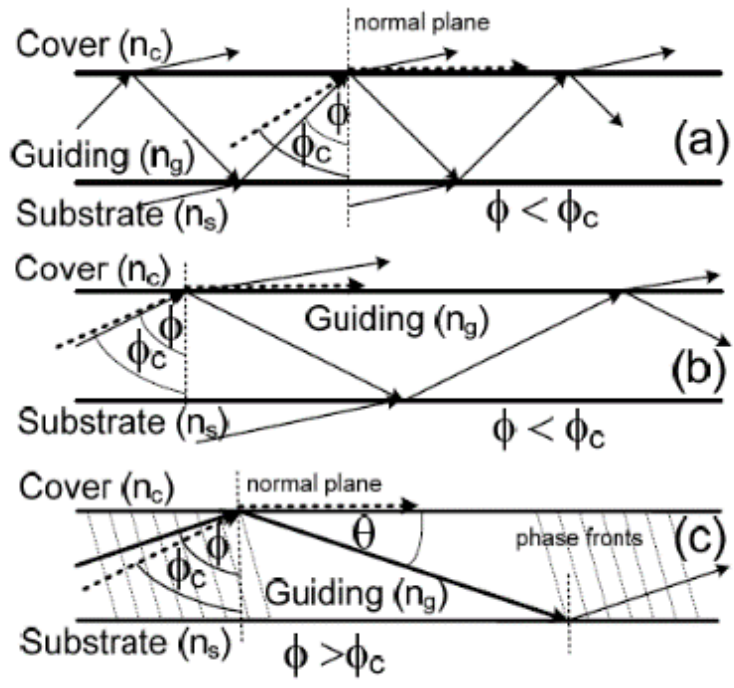

Figure 2. The ray picture of mode on an-symmetric step index slab waveguide [1]

\subsection{Optical Waveguide}

An optical waveguide is a physical structure that guides electromagnetic waves in the optical spectrum [1]. There are several types of waveguides that are used for optical signal transmission. Common types of optical waveguides include optical fiber and rectangular waveguides. Optical Waveguides are used as components of integrated optic circuit or as the transmission medium in local and long distance optical communication system.

Optical waveguides can be distinguished by its shape including planar, strip, and fiber, based on the structure of the modes single-mode and multi-mode, based on the distribution of refractive index step index and graded index, and based on its constituent materials glass, polymer, and semiconductor

\subsection{Normalization Value}

At the beginning of the explanation about the condition of the value of the propagation constant $\beta$ we see there is a value of $n_{1}>n_{\text {eff }}>n_{2}$ which can be written as follows:

$$
n_{\text {eff }}=\frac{\beta}{k_{0}}
$$

Where $k_{\ddot{0}}=2 \pi / \lambda$ from the explanation above we see also that the value of $n_{\text {eff }}$ lies between the values of $n_{1}$ and $n_{2}$, this is the characteristic of the TIR concept. Other parameters that are often used are normalization parameters of normalization constants which have a value between 0 and 1 that define with:

$$
b=\frac{n_{e f f^{-}-n_{2}^{2}}}{n_{1}^{2}-n_{2}^{2}}
$$

Other parameters that are often used are normalization frequencies $V$ defined by:

$$
V=k_{0} h \sqrt{n_{1}^{2}-n_{2}^{2}}
$$

\subsection{Wave Equations for Planar Wide}

Waveguides for wide waveguides where the dimensions are in the direction y is much larger than thickness, the field configuration along that direction remains around constant, see Figure 1. Therefore, we can consider only improvements in the $\mathrm{x}$-direction and set.

$$
\frac{\partial y}{\partial x}=0
$$

Also, the refractive index assumes only the $\mathrm{x}$-dependence

$$
\bar{n}=\bar{n}(x)
$$

\subsection{Temperature Effect in Refractive Index of Silica}

Temperature has effect to the material, the rising temperature make the electrons of material move faster. The thermo-optic effect is a phenomenon by which the refractive index of a substance changes with temperature [8]. In silica glass, this effect is characterized by an increase in the refractive index as the temperature rises. The Sell Meier coefficients at any temperature $\mathrm{T}$ are computed from the room temperature and the smoothed $d n / d T$ values by calculating the refractive indexes from the relation [5].

$$
n T=n R+(T-R)(d n / d T)
$$

Where $\mathrm{T}$ is the temperature in $0{ }^{\circ} \mathrm{C}, \mathrm{R}$ is the room temperature, $n T$ and $n R$ are the refractive index at $\mathrm{T}$ and at room temperature, respectively. $d n / d T$ is the thermos optic constant of the silica waveguide and the value of silica is $10^{-5}{ }^{\circ} \mathrm{C}[16]$

\subsection{Solution for the TE Mode Wave Equation}

Solution The general solution for the TE and TM wave equations for flat waveguides is essentially the difference in the application of the boundary conditions to the two wave equations. In the TM wave equation there is a factor $\left(n_{2}\right)$ in the continuity of the magnetic field and its derivative at the boundary between the material in the flat waveguide. TE mode uses an equation that involves $E_{y}$ and its derivatives. One observes that when $E_{x}=E_{z}=0$, from Eq. $H_{y}=0$. Equations describe them as modes

$$
\begin{gathered}
\beta E_{y}=-\omega \mu H_{x} \\
\frac{\partial H_{z}}{\partial x}+j \beta H_{x}=-j \omega \varepsilon E_{y} \\
\frac{\partial E_{y}}{\partial x}=-j \omega \varepsilon H_{z}
\end{gathered}
$$

The last step is to remove $H_{x}$ and $H_{z}$ using the second 
equation and finally eliminate $H_{x}$ using the first equation. We obtain the wave equation for TE mode

$$
\frac{\partial^{2} E_{y}}{\partial^{2} x}=\left(\beta-\bar{n}^{2} k^{2}\right) E_{y}
$$

Where $k=\frac{\omega}{c}=\omega \sqrt{\varepsilon_{0} \mu_{0}}$

\subsection{Solution for the TM Mode Wave Equation}

Procedure for finding the TM wave solution on a flat waveguide is exactly the same as the TE wave. The equation that describes TM mode is

$$
\begin{aligned}
\beta H_{y} & =\omega \varepsilon E_{x} \\
\frac{\partial H_{y}}{\partial x} & =j \omega \varepsilon E_{z} \\
\frac{\partial E_{z}}{\partial x}+j \beta E_{x} & =j \omega \mu H_{y}
\end{aligned}
$$

known that $\varepsilon=\varepsilon_{0} n_{2}$ and omits $E_{x}$ and $E_{z}$. From the first equation we specify $E_{x}$ and from the second equation we specify $E_{z}$. Changing the results into the third equation gives the wave equation for TM mode

$$
\bar{n}^{2} \frac{\partial}{\partial x}\left(\frac{1}{\bar{n}^{2}} \partial x \frac{\partial H_{y}}{\partial x}\right)=\left(\beta-\bar{n}^{2} k^{2}\right) H_{y}
$$

\section{Research Methodology}

The methodology used in this paper is the preparation of requirements, determination of specifications, study of literature, system design, system implementation, testing and system improvement, withdrawal analysis and conclusions.

\section{Calculation and Result}

The parameters used are: core width $h=5 \mu m$, wavelength range $=1.550 \mu m, \mathrm{n}_{\mathrm{c}}=1$ (refractive index in core), $\mathrm{n}_{\mathrm{f}}=1.50$ (refractive index in core), and $\mathrm{n}_{\mathrm{s}}=1.45$ (refractive index in cladding) or data shows in Table 1 below:

Tabel 1. Layer of waveguide

\begin{tabular}{cc}
\hline Reactive index & Thickness \\
\hline $\mathrm{n}_{\mathrm{c}=1} 1$ & Air \\
\hline $\mathrm{n}_{\mathrm{f}=1.50}$ & $5 \mu \mathrm{m}$ \\
\hline $\mathrm{n}_{\mathrm{s}=1.45}$ & - \\
\hline
\end{tabular}

By using the equation Solution TE and TM mode we get a Refractive index at $\mathrm{TE}_{0}=1.433$ and $\mathrm{TM}_{0}=1.438$, and by using the eq. (6), to analyze effect temperature at TE and TM mode, and the following figure describes that effective refractive index rises due to the temperature.

Figure 3 demonstrates the effective refractive index as a function of temperature. When the temperature is increased, the value of effective refractive index will be increased as well. These values of effective refractive index will be used for Modelling Temperature Effect on (Transverse Electric) TE mode Shape.

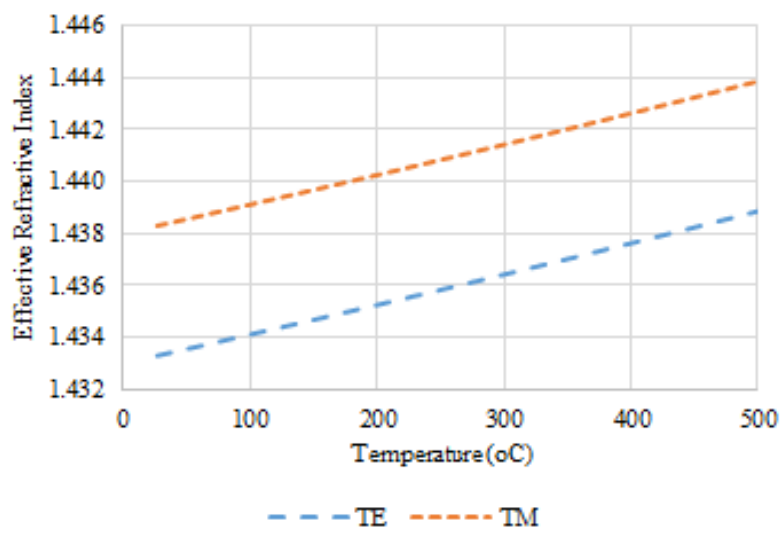

Figure 3. Refractive index as a function of temperature in ${ }^{0} \mathrm{C}$

From figure 4, the Electric Intensity takes the point between. It means that the highest Electric Intensity inside the core. Figure shows that there is an evanescent field outside the core of waveguide. It is the boundary between core and cladding with different wave motion properties because there is a refractive index difference between core and cladding.

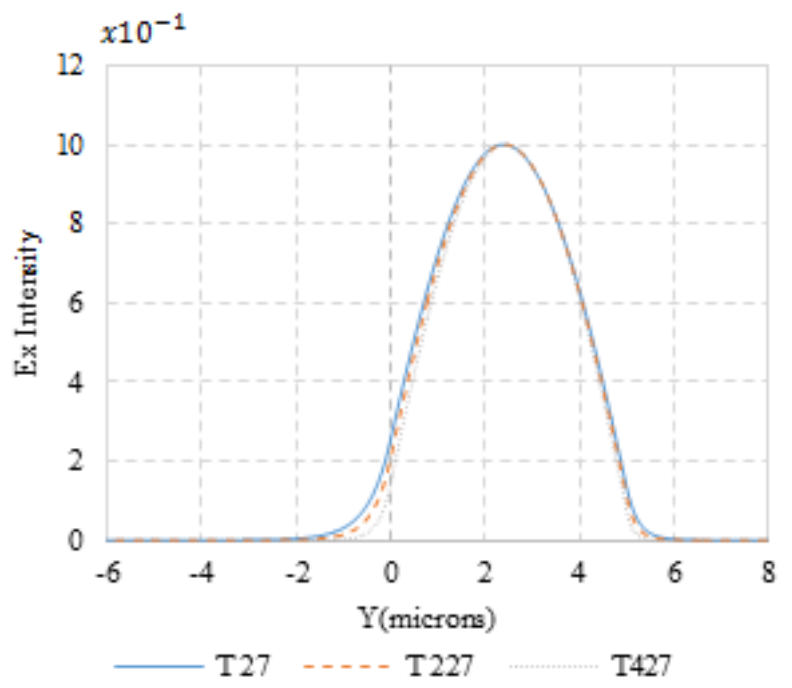

Figure 4. The Transverse Electric Mode Pattern

From figure 5, the Magnetic intensity takes the point between. It means that the highest Magnetic intensity propagates inside the core. Figure shows that there is an evanescent field outside the core of waveguide; it is the boundary between core and cladding with different wave motion properties because there is a refractive index difference between core and cladding. 


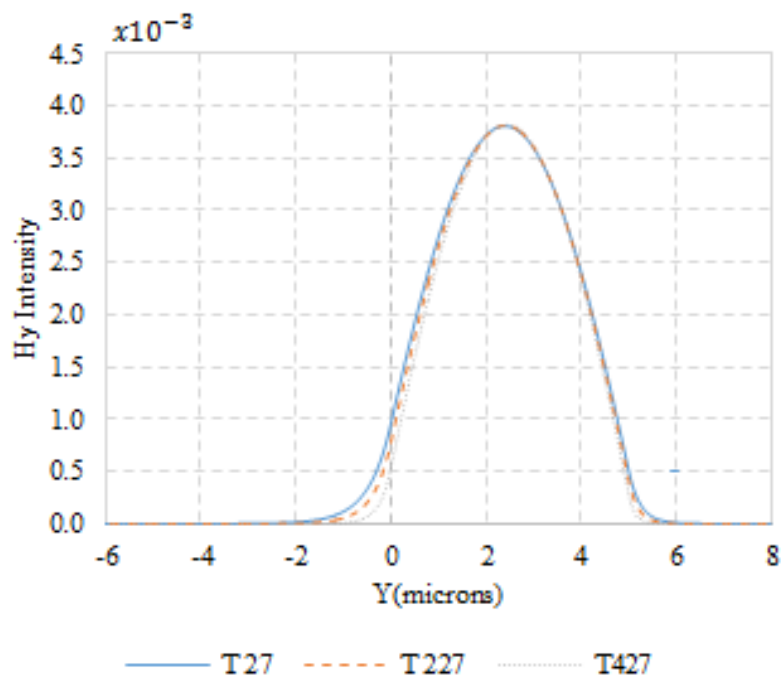

Figure 5. The Transverse Magnetic Mode Pattern

By using the same waveguide width, the light will not always confine in waveguide when we change the refractive index in core and cladding. It is because of the existence of evanescent field in waveguide become smaller. The bigger $n$, the bigger refractive index that causes the field propagation increasingly narrowed. And from value the Graph we know the value of a shifting core. So from the figure we describe the effect of temperature on the distance between modes in figure 6 .

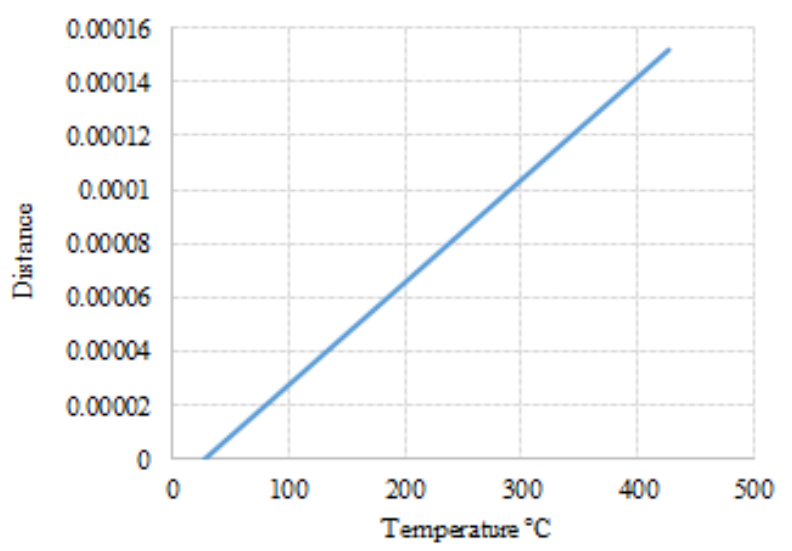

Figure 6. Graph of changes in temperature to distance

Figure 6 demonstrates the effective refractive index as a function of temperature. When the temperature is increased, the value of distance will be increased as well.

\section{Conclusions}

In this paper, an analytical solution was developed to estimate the effect of temperature on optical waveguides by analyzing the shape of TE \& TM mode. It was found that the temperature value affected the waveguide reactive index value but very small at range $27-427^{\circ} \mathrm{C}$. The higher the temperature value, the higher the refractive index value. As a result, the temperature sensitivity of the refractive index effectively affects the width of the core of the waveguide that can be seen from the effect of temperature on the width of the guide core. Therefore, it should be taken into account the effect of temperature on the shape of the value of TE \& TM mode to improve the performance of the waveguides to regions that have different temperatures.

\section{REFERENCES}

[1] Ooba, N., Hibino, Y., Inoue, Y., and Sugita, A., “Athermal silicabased arrayed-waveguide grating multiplexer using bimetal plate temperature compensator,” Electron. Lett. 36, 1800-1801 (2000)

[2] El-Khozondar, H., El-Khozondar, R., Shabat, M., Kock, A., "Stress effect on optical nonlinear waveguide sensor", J. Optical communication, 28 (3) in press.

[3] El-Khozondar, H. J. "Hydrostatic Stress Effect on the Optical Performance and the Stress Sensitivity of Optical Nonlinear Waveguide”, Annjah Journal for research (natural science), accepted (2007).

[4] El-Khozondar, H., Shabat, M., Abu Tair, G., and Abadla, M., "Thermal-stress effects on nonlinear thin film Waveguide Sensors," Journal of Physics \& Chemistry of Solid State 8 (2) 260-264 (2007).

[5] Huang, M., Yan, X., "Thermal-stress effect on the temperature sensitivity of optical waveguides", J. Opt. Soc. Am. B 20 (6), 1615- 1632 (2003).

[6] Boardman A., and Egan, P., "Optically nonlinear waves in thin films”, IEEE of quantum electronics, QE. 22 (2), 319-324 (1986).

[7] Stegeman, G., Seaton, C. T., Ariyasu, J., Wallis, R., Maradudin, A., "Nonlinear electromagnetic waves guided by a single interface”, J. Appl. Phys. 58 (7), 2453-2459 (1985).

[8] Huang, M., Yan, X., "Thermal-stress effect on the temperature sensitivity of optical waveguides”, J. Opt. Soc. Am. B 20 (6), 1615-1632 (2003). 